\title{
Varying Load Detection in A Gearbox System Based on Adaptive Threshold Estimation
}

\author{
A. Kouadri ${ }^{1, a}$, G. R. Ibrahim ${ }^{2}$ and A. Albarbar $^{3}$ \\ ${ }^{1}$ Signals and Systems Laboratory, Institute of Electrical and Electronics Engineering, University of Boumerdes, \\ ${ }^{2}$ Department of Mechanical Engineering, College of Engineering, University of Anbar, Iraq. \\ ${ }^{3}$ School of Engineering, Manchester Metropolitan University, ManchesterM1 5GD, UK.
}

\begin{abstract}
This article presents an adaptive threshold computation for varying load detection in gearbox system. This method was applied on the vibration signal which is captured from gearbox housing. The experimental work was carried out using a three-stage gearbox connected with a motor and a generator. The developed adaptive threshold is obtained through several repeated experiments in the healthy mode and under the same operating conditions. An appropriate statistical test is used to examine the validity of the adaptive threshold estimation approach. Besides and for all experiments, a confidence interval is obtained which is closely linked to the distribution frequencies of the gearbox vibration signal as a random variable. In addition, several significance levels are considered to show the performances of the proposed adaptive thresholding technique compared to the limitations of the fixed threshold through the rate of false detection alarms. It is demonstrated from various experimental varying load mode of a gearbox system the effectiveness and accuracy of the adaptive threshold in term of evaluating gearbox operating conditions.
\end{abstract}

\section{Introduction}

Gearboxes fault diagnosis based on vibration measurements have grown significantly due to the progress in digital signal processing algorithms and the implementation techniques. A great deal of effort has been put into the development of monitoring approaches for such faults within mechanical transmission systems. For instance, Belsak et al[1] studied fatigue crack in tooth roots of the spur gear, using it to conduct the finite element method (FEM) to simulate fatigue crack growth and short- time fourier transformation (STFT). Kara et al applied advanced signal-processing techniques, such as the discrete wavelet transform (DWT) and a corrected multi-resolution Fourier transform (MFT), to investigate the vibration and current transients in a multistage gearbox under transient load [2]. Wu et al [3] studied the effect of tooth crack on the vibration response of a one-stage gearbox with spur gear. The vibration response of a pair of meshing gears was simulated by a lumped parameter model. They used several statistical indicators to reflect the change in the vibration response caused by the tooth crack. Ibrahim and Albarbar [4] presented a comparison between empirical mode decomposition (EMD) and smoothed pseudo-Wigner-Ville distribution (SPWVD) methods based on vibration signature and energy calculation procedure for monitoring gearbox systems. This article illustrates

${ }^{\text {a }}$ Corresponding author : a_kouadri@hotmail.com 
that the calculation of energy using EMD techniques offers a more effective way to detect early faults than the SPWVD method, and that the computation of energy using the EMD technique is faster than the SPWVD method.

In this work, the gearbox vibration signal is used as a varying load detection index. This detection is a test of the adherence of the gearbox vibration signal to the confidence interval which is obtained after several independent experiments which are conducted in the same functioning conditions. The accuracy and effectiveness of the vibration signal-based approach for varying load detection are based on the powerful adaptive confidence interval which quantifies the noises in and on the considered system. More specifically, an appropriate test statistics based on the statistical characteristics of the analysed signal is used to define the best random phenomena associated with it and examine the validity of the proposed fault detection approach.

This paper is organized as follows: the exploitable experimental database is firstly presented in section 2 . Then, a qualitative statistic analysis of the available database is carried out by giving some statistical quantities. In section 4 , the proposed procedures are presented in a general manner to estimate the adaptive threshold. The use of these procedures to overcome the problem of uncertainties in measurement and detection with accuracy of varying loads in the gearbox is reported in section 5 in which the best results achieved are discussed and, finally, some conclusions are drawn in section 6 .

\section{Test rig and baseline data}

The experimental work was carried out using a three-stage helical gearbox connected with a motor and a generator as shown in figure 1 . Tests were carried out for a healthy pair of gears under varying loads $(0 \%, 20 \%$ and $80 \%$ of the total load). The drive pinion at the first stage had 40 teeth meshing with a 20-tooth wheel. The pinion gear at the second stage had 80 teeth meshing with 20 -tooth wheel. The pinion gear at the third stage had 80 teeth meshing with 32-tooth wheel. The vibration signals were collected using an accelerometer mounted vertically on the gearbox housing. The accelerometers were B\&K type 4371 with sensitivity of $10 \mathrm{mV} / \mathrm{g}$ and suitable for vibration measurements within a range of $1 \mathrm{~Hz}$ to $12 \mathrm{kHz}$. The sampling frequency was set to $5 \mathrm{kHz}$. Before the accelerometer signals were fed to the analogue-to-digital converter NI USB 9233 card, they passed through a B\&K type 2635 charge amplifier to condition the signal. The charge amplifier converts the accelerometer high impedance, low charge (in the range of Pico-coulomb) signal into low impedance and high voltage (in the range of $\mathrm{mV}$ ) and the cut-off frequency for filter initialisation was set to $10 \mathrm{kHz}$.

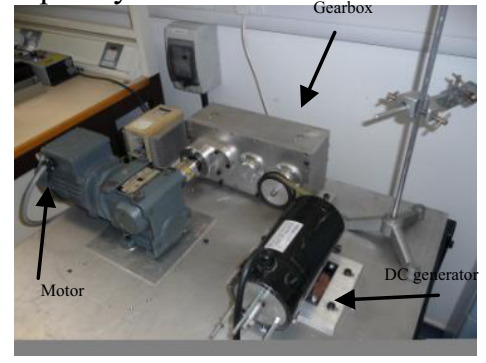

Figure 1. Gearbox test rig

In this paper, the database was divided into $N=66$ parts, recorded from $N$ different repeated experiments. These experiments were conducted in a healthy mode and under the same operating conditions. Each database part includes $T=400$ samples. Corresponding to 0.08 seconds with reading frequency equals 20 milliseconds. These database parts are organized in a matrix $\boldsymbol{S}$ of 66 rows and 400 columns.

\section{A statistical and qualitative analysis of vibration signals}


In the present section, a preliminary statistical analysis of the database is carried out. Figure 2 reports the instantaneous statistical parameters of the database. High-order statistics are also illustrated and can be used as a reference value for adaptive threshold estimation performance [5] (figure 2). At each sample time, we can determine the mean of the variable

$$
M(t)=\frac{1}{N} \sum_{i=1}^{N} S_{i}(t)
$$

Also, we can compute the other instantaneous statistical parameters as follow:

$$
\begin{gathered}
V(t)=\frac{1}{N} \sum_{i=1}^{N}\left(S_{i}(t)-M(t)\right)^{2} \\
S k(t)=\frac{1}{N} \sum_{i=1}^{N}\left[\frac{\left(S_{i}(t)-M(t)\right)}{\sqrt{V(t)}}\right]^{3} \\
K u(t)=\frac{1}{N} \sum_{i=1}^{N}\left[\frac{\left(S_{i}(t)-M(t)\right)}{\sqrt{V(t)}}\right]^{4}
\end{gathered}
$$

where $V(t), S k(t)$ and $K u(t)$ represent respectively the variance, skewness and kurtosis at sample time $t$.
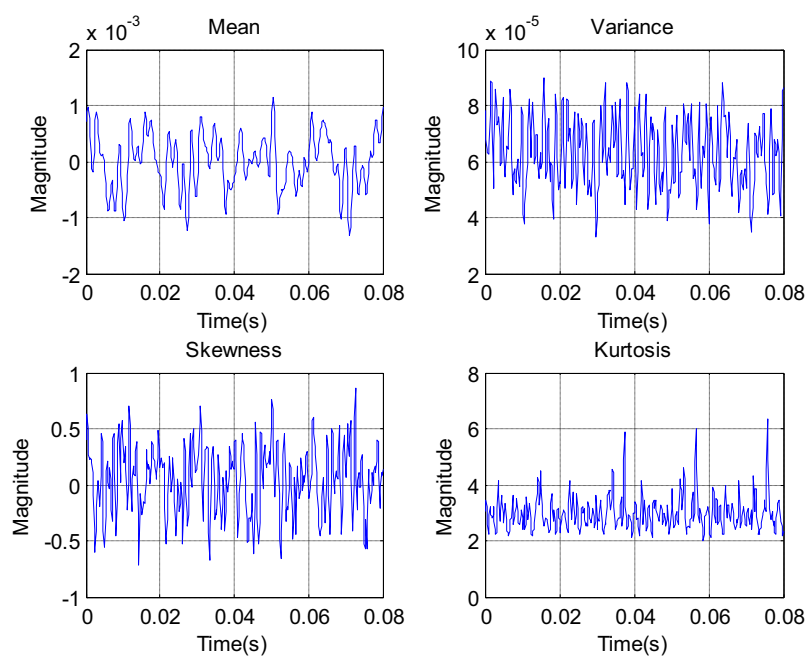

Figure 2. Different instantaneous statistical parameters of a gearbox vibration signal for repeated experiments

We focused on the two first statistical parameters, because it can be seen a large variation in the mean which indicates the non homogeneity of the vibration signal. The variance confirms that it is strongly related to the noise on the measurement device. Therefore, the vibration signal is a non stationary process. In addition, $S(j)$ at the sample time $j$ is considered as a random variable for which we can find its probability distribution law. By looking at the histograms make randomly for several sample time and their corresponding skewness and kurtosis parameters, it seems that a normal distribution well describes its distribution [6] (figure 3). 

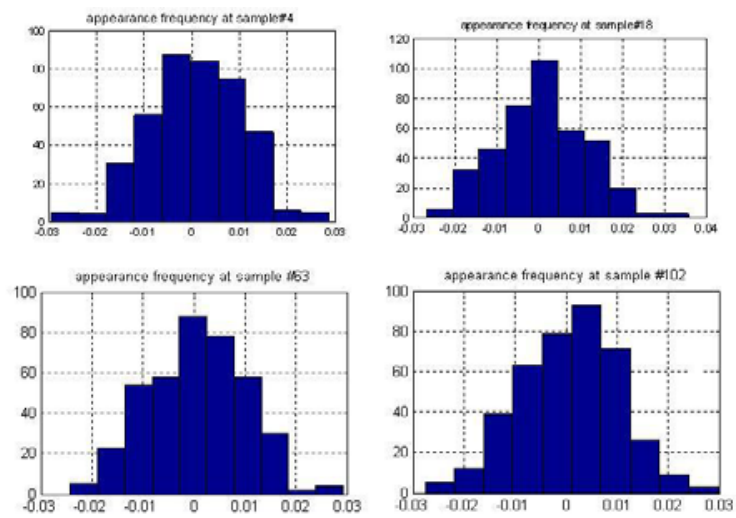

Figure 3. Different histograms of vibration signal at different sample time.

\section{Adaptive threshold computation}

The measurements are corrupted by noise, and, more importantly the characterization of signal's dispersion in the presence of faults, random factors, and disturbances on the system is difficult to interpret within a quantitatively perfect threshold [7]. Based on the fact that the vibration signal of the gearbox changes from one experiment to another under the same operating conditions, the sampled vibration signal is classified as a random variable $\Sigma(t)$. Thus, for each experiment, an instantaneous vibration signal $S_{i}(t), i=1, \ldots, N$, is evaluated. To increase the sensitivity of the fault detection index based on vibration signal $S(t)$ at each sample time $t$, an adaptive threshold is adopted in this study. Specifically, it should be constructed, from a given deduction, a random interval containing $S_{i}(t)$ with an acceptable probability. This probability will define a confidence level. In practice, the confidence level $(1-\alpha)$ is typically selected to be $95-99 \%$. Considering the centred interval in $S_{i}(t)$, i.e. $] S_{i}(t)-\varepsilon(t), S_{i}(t)+\varepsilon(t)[, \varepsilon(t)$ is determined as:

$$
P\left(S_{i}(t)-\varepsilon(t) \prec \hat{S}_{i}(t) \prec S_{i}(t)+\varepsilon(t)\right)=1-\alpha
$$

where $\alpha$ represents the error risk incurred that $S_{i}(t)$ is located in the considered interval. The parameter $\varepsilon(t)$ depends on the value of the error risk $\alpha$ and the distribution law associated to the random variable $\Sigma(t)$, so:

$$
\varepsilon(t)=F^{-1}(1-\alpha) \sqrt{V(t)}
$$

where $F^{-1}($.$) represents the inverse of the probability distribution function value. Therefore, the$ adaptive threshold limits at a confidence level $(1-\alpha)$ for which the confidential condition of the equation (5) verifies, are given by the confidential interval, so:

$$
\left.C I_{\alpha}(t)=\right] M(t)-F^{-1}(1-\alpha) \sqrt{V(t)}, M(t)+F^{-1}(1-\alpha) \sqrt{V(t)}[
$$


For the purpose of adaptive thresholding, it is required to evaluate the confidence interval at each sample time $t$ using equation (7). A fault in a system can be detected, if a sampled vibration signal is outside the corresponding confidence interval.

The probabilistic computation of the fault detection index based on the gearbox vibration signal $S(t)$ and its associated adaptive threshold given by the confidence interval is based on the fact that the random variable $\Sigma(t)$ follows a specified distribution law of mean $M(t)$ and variance $V(t)$ [8]. This hypothesis should apply to all sequences of the vibration signal and at each sample time. The skewness and kurtosis indicate automatically the normality distribution of the phenomena under study (See figure 2 and 3). In the normal distribution case, the skewness and kurtosis are close to 0 and 3 , respectively. However, the skewness is an asymmetric distribution measure of the random variable $\Sigma(t)$ around its mean $M(t)$, the skewness defined by equation 3 is normally distributed, such that:

$$
S k(t) \propto \mathcal{N}\left(0, \sqrt{\frac{6}{N}}\right)
$$

The kurtosis measures the pointed or flat characteristics of the $\Sigma(t)$ distribution law. It follows:

$$
K u(t) \propto \mathcal{N}\left(3, \sqrt{\frac{24}{N}}\right)
$$

At a given sample time $t$, the random variable $\Sigma(t)$ obeying a normal distribution law $\mathcal{N}(M(t), \sqrt{V(t)})$ if the two quantitative statistics measurement $S k(t)$ and $K u(t)$ follow jointly a centered and reduced normality distribution $\mathcal{N}(0,1)$, respectively.

\section{The adaptive thresholding performances}

The adaptive threshold for the vibration signal of the gearbox is calculated from 66 trajectories under the hypothesis that the given sampled vibration signal $S(t) \propto \mathcal{N}(M(t), \sqrt{V(t)})$ with different significant values. The efficiency of the detection algorithm is based on the computation of the confidence intervals which provide an estimate of the amount of error involved in the considered data and characterize the precision of the computed statistical estimates. The Skewness-Kurtosis nonparametric test validates the normal distribution law, for a typical confidence level of $95 \%$, of the vibration signal with acceptable probabilities ( $p$-value equals 0.857 ).

The probability, of observing given sampled gearbox vibration signal of all experiments under the assumption that this later is normally distributed, is computed and evaluated. Furthermore, the normally distribution is justified to an appropriate satisfaction probability degree. However, the chosen significance level is related to the degree of certainty you require in order to reject that the sampled gearbox vibration signal follows normal law. This is true if the probability of observing sampled result is less than the significance level. Therefore, the vibration signal of the gearbox is fully satisfied to be normally distributed with fairly acceptable jointly probabilities of skewness and kurtosis. These obtained probabilities are at almost samples closed to 1 .

To evaluate the performance of the detection scheme based upon the adaptive threshold; several cases, a healthy pair of gears under varying loads $(20 \%$ and $80 \%$ of the total load), are examined. Figure 6 illustrates the vibration signal variation at different loads with the confidence interval limits which are developed at the confidence level $99 \%$. It can be seen clearly that the gearbox vibration signal is outside the confidence interval limits. Therefore, the varying load is accurately detected using the adaptive thresholding technique. Regarding the amplitude of the gearbox vibration signal which becomes quite small at high load, the adaptive threshold indicates well the occurrence of the varying load in spite of the confidence interval is quite large at the confidence level $99 \%$. Consequently, the results confirm the high sensitivity and accuracy of the adaptive threshold in detecting varying load. 

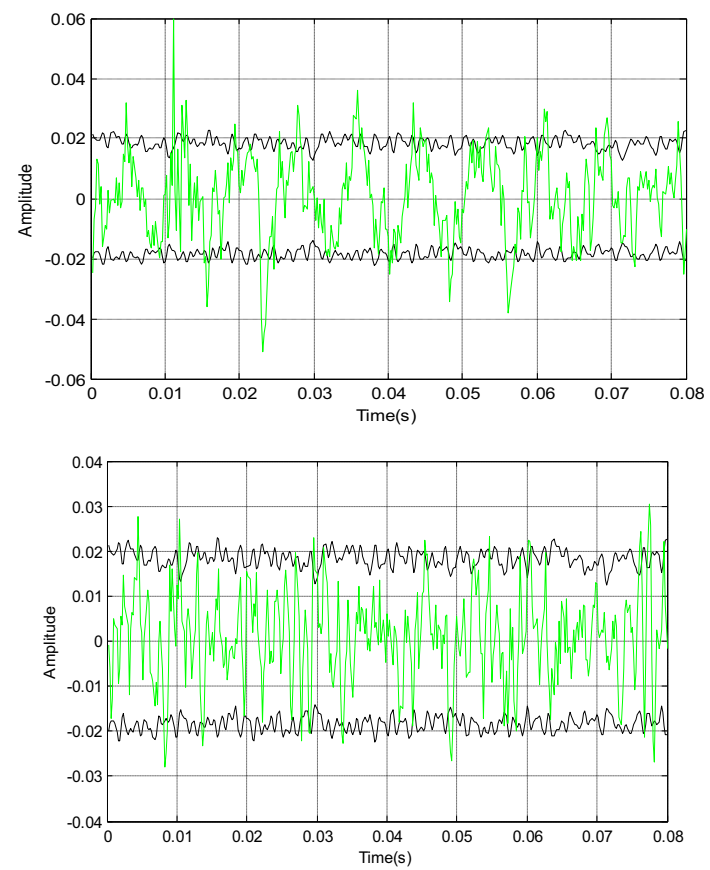

Figure 4. Load detection ( $20 \%$ and $80 \%$ load, respectively)

\section{Conclusion}

The present paper deals with one of the major problems that arise when searching for a reliable, accurate, and easily exploitable, adaptive threshold, which can indicate with accuracy the presence of a varying load in the gearbox. The developed adaptive threshold is based on the vibration signal measurement for which it is able to overcome the problem of false detection and non detection of a fault by using the fixed thresholding techniques. The obtained results also illustrate that under different situations the adaptive threshold can accurately detect changes in the experimental, threestage gearbox connected with motor and generator, variables. Furthermore, the principle of calculation of confidence interval at different confidence level, after testing the distribution law of the database using Skewness-Kurtosis method, is sufficient to constitute an interesting and helpful tool to overcome the difficulties of parametric uncertainties quantification and devices measurement inaccuracies which increase the number of false alarms. It is also important to note the high sensitivity of the adaptive threshold in detecting varying loads with fairly low amplitudes in gearbox vibration signal.

\section{References}

1. Belsak, A. and J. Flasker. Detecting cracks in the tooth root of gears. Engineering Failure Analysis 14 (2007) 1466-1475.

2. Kara, C. and A. Mohanty. Vibration and current transient monitoring for gearbox fault detection using multiresolution Fourier transform. Journal of Sound and Vibration 311 (2008) 109-132.

3. Wu, S., M. Zuo and A. Parey. Simulation of spur gear dynamics and estimation of fault growth. Journal of Sound and Vibration 317 (2008) 608-624 .

4. G. R., Ibrahim and A., Albarbar. Comparison between Wigner-Ville distribution- and empirical mode decomposition vibration-based techniques for helical gearbox monitoring. Proc. IMechE 
Part C: J. Mechanical Engineering Science. Published online 16 June 2011. DOI: $10.1177 / 0954406211403571$

5. Zhijian Wang, Zhennan Han, Fengshou Gu, James Xi Gu and Shaohui Ning, A novel procedure for diagnosing multiple faults in rotating machinery, ISA Transactions 55 (2015) 208-218,

6. Lotfi Saidi, Jaouher Ben Ali and Farhat Fnaiech, Application of higher order spectral features and support vector machines for bearing faults classification, ISA Transactions 54 (2015) 193-206,

7. Vicenç Puig, Saúl Montes de Oca and Joaquim Blesa. Adaptive threshold generation in robust fault detection using interval models: time-domain and frequency-domain approaches, International Journal of Adaptive Control and Signal Processing, 27 (10) (2013) 873-901.

8. Joanes DN, Gill CA. Comparing measures of sample skewness and kurtosis. Journal of the Royal Statistical Society, (Series D): The Statistician 47 (1) (1998) 183-189. 